\title{
Shape of Melting Ice Depends on Temperature
}

\author{
Experiments reveal that the shape of submerged, melting ice depends on \\ temperature, suggesting that natural ice structures can provide clues \\ about water temperatures.
}

By Mark Buchanan

ce cylinders submerged in water form one of three distinct structures as they melt, depending on the temperature

of the surrounding water, according to new experiments [1]. Researchers found that the temperature-dependent buoyancy of meltwater determines the shape. As our warming world suffers a progressive loss of natural ice, an understanding of the effects of temperature on ice shapes may help researchers infer environmental conditions from observations of ice structures.
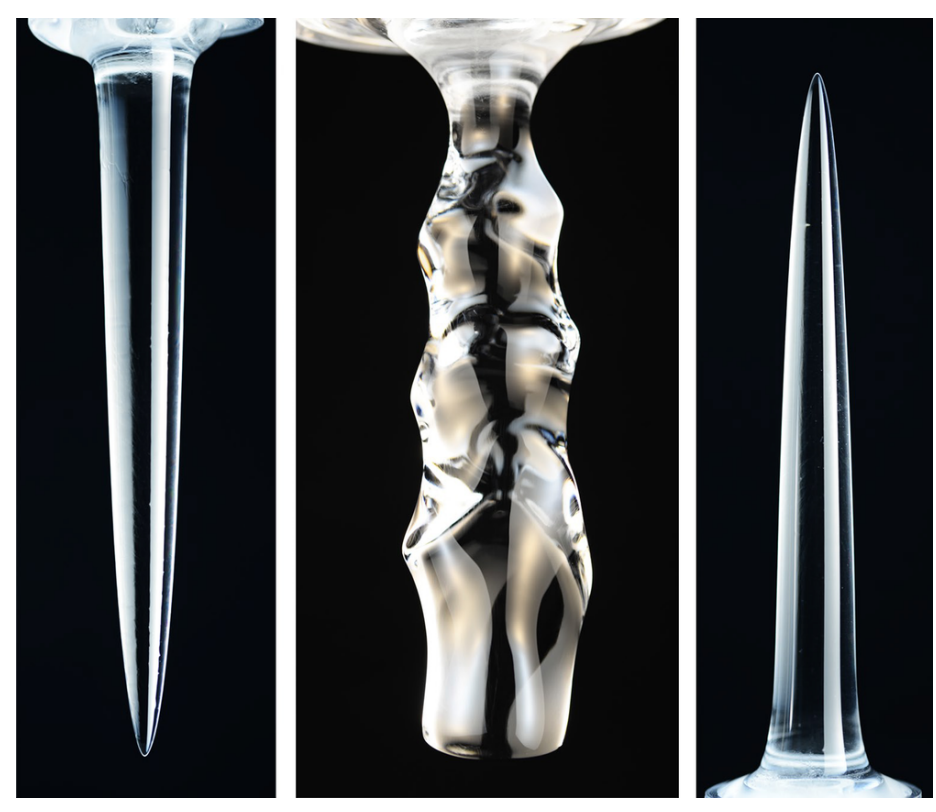

Running hot and cold. The temperature of the water bath determines the shape of a melting cylinder of ice: $4{ }^{\circ} \mathrm{C}$ (left), $5.6^{\circ} \mathrm{C}$ (center), and $8{ }^{\circ} \mathrm{C}$ (right).

Credit: S. Weady et al. [1]
Water is highly unusual among liquids in that its density takes a maximum value at a particular temperature, $4^{\circ} \mathrm{C}$, well above its freezing point. So as water cools below $4^{\circ} \mathrm{C}$, rather than contracting, it expands, which explains why ice floats on water. This property of water is well known, but no one has previously looked at its effects on the shape of melting ice. And melting ice is an important subject in a warming global climate.

Leif Ristroph of New York University and colleagues immersed cylindrical ice samples $15-20 \mathrm{~cm}$ long and a few centimeters in diameter in water at a temperature between $2{ }^{\circ} \mathrm{C}$ and $10^{\circ} \mathrm{C}$. The cylinders were fixed in a vertical orientation. During melting, the researchers allowed the water temperature to cool down in the zone near the ice but held the temperature fixed far away.

Three distinct temperature regimes led to distinct results. For water at $4{ }^{\circ} \mathrm{C}$ or colder, the cylinder gradually melted away from below, leaving a smooth, inverted pinnacle (a downward-pointing, cone-like shape). For temperatures between $5^{\circ} \mathrm{C}$ and $7{ }^{\circ} \mathrm{C}$, a more complex undulating pattern appeared on the melting cylinder. Finally, for temperatures above $7^{\circ} \mathrm{C}$, the ice formed an upward-pointing pinnacle.

Ristroph and colleagues suspected that the various shapes might be explained by differences in the buoyancy of the water produced by the melting ice. To test this idea, the team ran a series of simulations, which confirmed their suspicions.

For bath temperatures around $4{ }^{\circ} \mathrm{C}$, the simulations showed that the colder liquid coming from the melting ice-being less dense than the surrounding liquid-rises. This upward flow along the ice surface pulls in relatively warmer water at the 


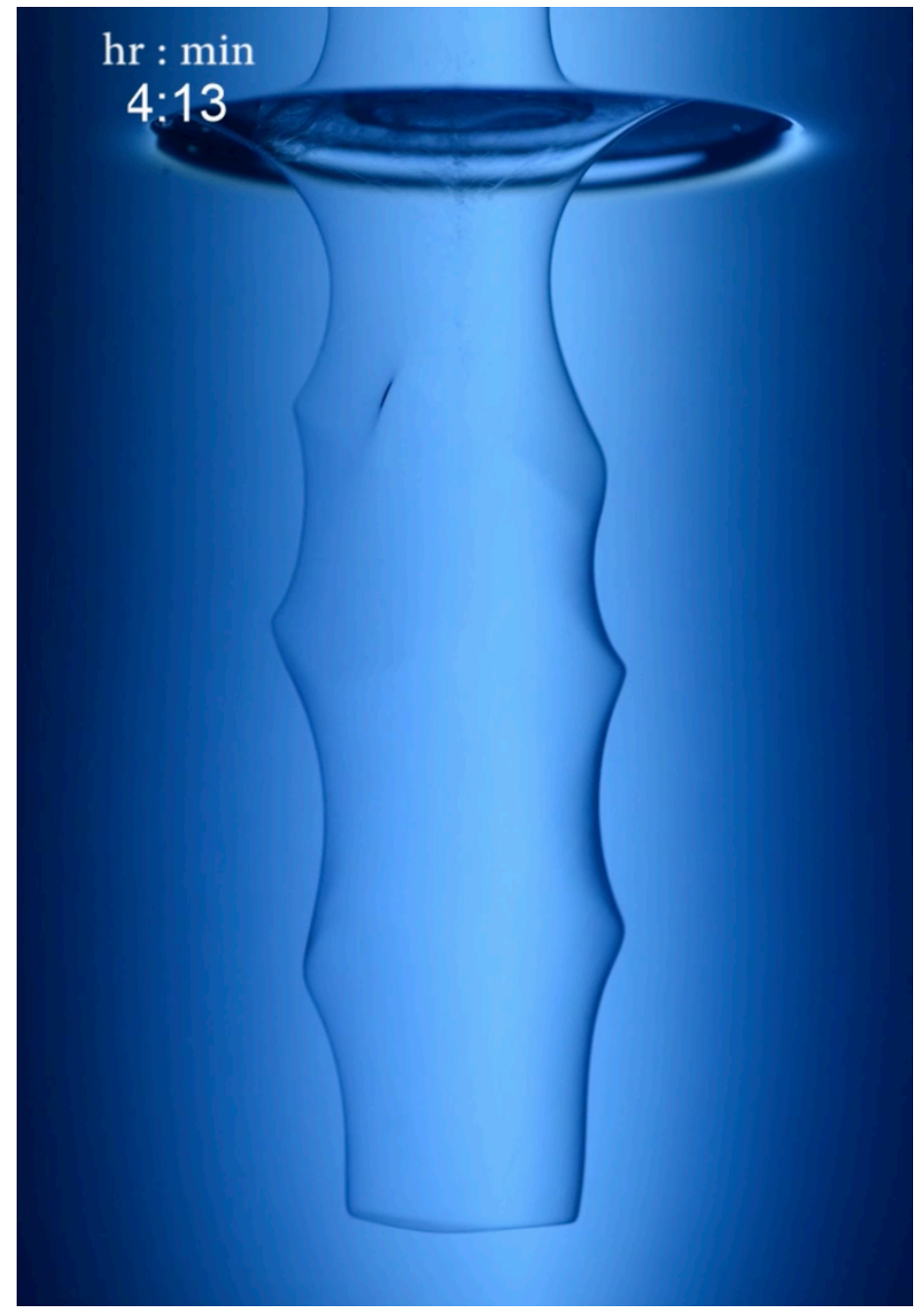

A submerged cylinder of ice melts at the intermediate temperature of $5.6^{\circ} \mathrm{C}$. Contrasting flows-an upward flow close to the ice, and a downward flow a bit farther away-set up a series of convection rolls that produce a wavy pattern on the ice surface.

Credit: S. Weady et al. [1]

bottom of the cylinder, so melting at that location is the most rapid. As water flows upward, the ice cools it, causing the melting rate to be slowest at the top of the cylinder. This pattern of disparate melting rates leads to a downward-pointing pinnacle. In contrast, the team found that the opposite happens for sufficiently warm water temperatures. The cold liquid near the ice surface is denser than that far away and sinks, drawing in warmer water from above, melting the upper zone faster, and causing the upright pinnacle to form.

For intermediate temperatures, the team found, the situation is more subtle, since the meltwater is likely denser than surrounding water in some places and less dense in others, leading to more complex flows. The simulations showed that colder, less dense water near the ice surface flows upward, while warmer, denser water, millimeters away, flows downward. A vertical row of vortices forms as these opposing flows move past each other. These "convection rolls" carve the wave-like pattern of melting because they bring warmer liquid against the ice surface.

Ristroph suggests that these results may offer some help to scientists who interpret shapes and patterns seen in natural ice. "Our findings offer previously overlooked mechanisms for the formation of pinnacles and waves," he says, "both of which are commonly observed on icebergs and other natural ice structures."

"I'm flabbergasted by this work," says fluid physicist Detlef Lohse of the University of Twente in the Netherlands. "All these patterns only arise due to the density anomaly of water and therefore only in a small window of temperatures. I wouldn't have expected these pinnacle shapes." Lohse says the work "has tremendous relevance" for studies of melting icebergs and glaciers because the melting rate is strongly affected by the fluid flow pattern. Some of the basic physics principles are still not well understood, he says, but "Ristroph's paper elucidates this physics in a wonderful way."

Mark Buchanan is a freelance science writer who splits his time between Abergavenny, UK, and Notre Dame de Courson, France.

\section{REFERENCES}

1. S. Weady et al., "Anomalous convective flows carve pinnacles and scallops in melting ice," Phys. Rev. Lett. 128, 044502 (2022). 